Innovación y Desarrollo Tecnológico y Social (2019) 1 (1): 61-72

\title{
Protocolo para la propagación por minicepas y miniestacas de yerba mate (Ilex paraguariensis St. Hil.)
}

\author{
Rocha $^{1,3}$, P.; Duarte1, E.; Gortari, 2, F.; Morales ${ }^{1}$, V. y Niella' ${ }^{1}$ F. \\ ${ }^{1}$ Facultad de Ciencias Forestales-UNaM \\ ${ }^{2}$ Becario Posdoctoral CONICET \\ 3 procha910@gmail.com
}

\begin{abstract}
Resumen. La domesticación de la yerba mate (Ilex paraguariensis St. Hil) es muy reciente, razón por la cual, las plantaciones son heterogéneas, presentan plantas de diferentes tamaños, crecimiento y vigor, resistencia a enfermedades y tipo de hojas entre otras. Esta variación se debe fundamentalmente a que las plantas son multiplicadas por semillas y en consecuencia ocurre una gran segregación de caracteres. A su vez, la producción de plantines de yerba mate desde semillas presenta una serie de limitantes: bajo porcentaje de germinación (13\% a 14\%), largo periodo para el comienzo de la germinación (100 y 365 días), las semillas de calidad genética son escasas y caras, se debe realizar el trasplante al contenedor final, elevado costo en mano de obra y largo período de viverización (12 a 15 meses).
\end{abstract}

La técnica de propagación vegetativa por minicepas y miniestacas, desarrollada y descripta en el presente trabajo, es una alternativa a la producción de plantines por semillas, dado que permite aumentar la producción de propágulos (miniestacas) por unidad de superficie, con un menor tiempo de viverización. Además, esta técnica puede ser adaptada a la realidad de los pequeños y medianos productores.

El objetivo del proyecto fue desarrollar una metodología de propagación vegetativa de yerba mate, que maximice el uso del espacio en el vivero, aumente la tasa de multiplicación y homogeneidad en el manejo de vivero, factible de ser implementada por pequeños y medianos viveristas.

Multiplicación clonal; semillas;propagación vegetativa; viveros; productores

Recibido: 04/06/2019 Aceptado: 09/08/2019

DOI: https://doi.org/10.24215/26838559e005

\section{Yerba mate (I/ex paraguariensis St. Hil.) ministumps and minicuttings clonal propagation protocol}

\begin{abstract}
Domestication of yerba mate (Ilex paraguariensis St. Hil) is very recent, therefore, the plantations are heterogeneous, presenting plants of different sizes, growth and vigor, resistance to diseases and type of leaves among others. This variation is mainly due to the fact that plants are producedfrom seeds and consequently a high segregation of characters occurs. At the same time, plant production fromseedspresents low percentage of germination (13\% to $14 \%$ ), long period for the beginning of the germination (100 and 365 days), high labor cost and
\end{abstract}




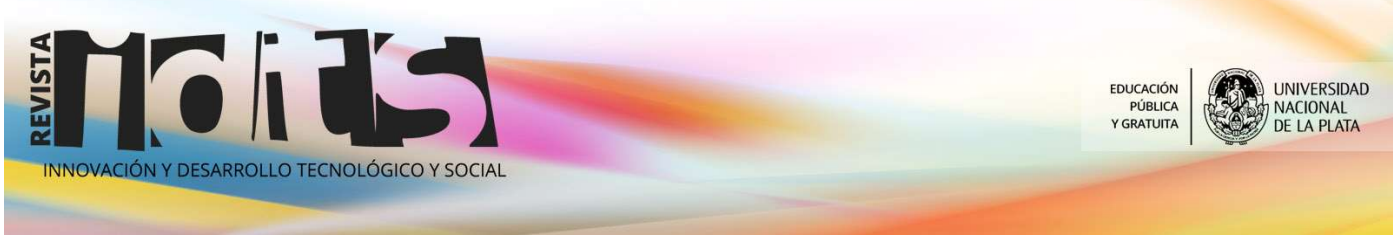

Innovación y Desarrollo Tecnológico y Social (2019) 1 (1): 61-72

long period of nursing (12 to 15 months), andseed with genetic quality is scarce and expensive.

The technique of vegetative propagation by ministumps and minicuttings, developed and described in the present work, is an alternative to the production of seedlings, since it allows to increase the production of propagules (minicuttings) per unit area, with a shorter time of nursing. In addition, this technique can be adapted to the reality of small and medium nurseries.

The objective of the project was to develop a methodology of vegetative propagation of yerba mate, which maximizes the use of space in the nursery, increases the multiplication rate and homogeneity in nursery management; feasible to be implemented by small and medium nurserymen.

Clonal multiplication; seeds; vegetative propagation; nurseries; farmers

\section{Novedad u originalidad local en el conocimiento:}

El género Ilex (Aquifoliaceas) se distribuye en todos los continentes, particularmente en regiones templadas, tropicales y subtropicales. Una de las especies más importantes, dentro del género, es Ilex paraguariensis St. Hil, conocida como yerba mate, propia del noroeste de Argentina, sur de Brasil y este de Paraguay. Sus hojas se consumen en forma de infusión (mate y te) (Eibl et al., 2000). La yerba mate constituye uno de los principales cultivos de Misiones. Según datos del INYM(2016), existen 160.000 hectáreas cultivadas. Representando una fuente importante de trabajo, con potencial para proveer servicios ambientales, como el secuestro de carbono.

El cultivo de yerba mate se inició en 1930, en monocultivos puros y con cubierta arbórea, en sistemas agroforestales (Eibl et al., 2000), previamente se extraía directamente del monte nativo. Su domesticación es muy reciente, razón por la cual, las plantaciones son heterogéneas, presentan plantas de diferentes tamaños, crecimiento y vigor, resistencia a enfermedades y tipo de hojas entre otras. Esta variación se debe fundamentalmente a que las plantas son multiplicadas por semillas y en consecuencia ocurre una gran segregación de caracteres.

La técnica de propagación vegetativa por minicepas y miniestacas, es una alternativa a la producción de plántulas por semillas, dado que permite aumentar la producción de propágulos (miniestacas) por unidad de superficie, con un menor tiempo de viverización. Además, esta técnica puede ser adaptada a los sistemas de producción de los pequeños y medianos productores para generar plántulas con caracteres deseables de yerba mate.

\section{Grado de relevancia:}

La multiplicación por semilla de la yerba mate presenta varias limitaciones tales como bajo porcentaje de germinación (13\% a 14\%), obteniéndose de esta manera, aproximadamente entre 20.000 a 22.000 plántulas/kg de semilla (Prat Kricun, 1993). El 


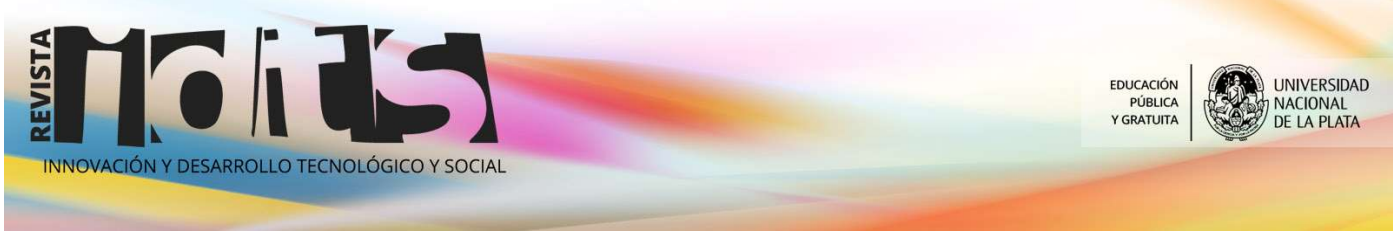

Innovación y Desarrollo Tecnológico y Social (2019) 1 (1): 61-72

periodo requerido para el comienzo de la germinación es largo, entre 3 y 9 meses (Galíndez et al., 2018), hecho que demanda la siembra en almácigo, con los costos y superficie que esto implica (costo de oportunidad). A esto se debe sumar la necesidad de trasplante al contenedor final; mayor costo en mano de obra y largo período de viverización, de 12 a 15 meses. Aun cuando existen instituciones nacionales que disponen de semilla provenientes de huertos policlonales con alto grado de mejora genética, el costo del kilogramo de semilla es alto y la disponibilidad es baja y variable. La propagación vegetativa, a través de la técnica de macropropagación por minicepas y miniestacas de yerba mate, representa una oportunidad para solucionar las limitaciones que tiene la reproducción sexual; aumentar la disponibilidad de material vegetal y permitir a todos los viveros regionales contar con material de alta calidad genética, beneficiando en forma directa a los pequeños productores, quienes podrán maximizar sus rendimientos, al aumentar sus ingresos por superficie plantada, acompañado por buenas prácticas agrícolas y mejorando de esta manera su calidad de vida.

\section{Grado de pertinencia:}

La propagación vegetativa mediante la tecnología de minicepas y miniestacas fue desarrollada para las plantas del género Eucalyptus (Assis y Mafia, 2007), y en especies nativas de Misiones, como son: Peltophorum dubium, Cordia trichotoma, Myrocarpus frondosus, Helietta apiculata, Cedrela fissilis, Eugenia involucrata (Niella et al., 2014b; Ramirez y Rocha, 2017; Rocha et al., 2015). En yerba mate, ha sido estudiada desde hace más de una década, donde se ha evaluado como influyen diferentes factores como el sustrato, el ambiente, la aplicación de hormonas, la época y número de cosechas en la capacidad de brotación, en la producción de miniestacas y en la capacidad de enraizamiento (Bitencourt, Zuffellato-Ribas, Wendling y Koehler, 2009; Brondani, Araujo, Wendling y Kratz, 2008; Brondani et al., 2009; Naumann, Rocha, Duarte, Morales y Niella, 2017; Niella et al., 2014a; Pino y Laviosa, 1998; Wendling, Dutra y Grossi, 2007; Wendling, Trueman y Xavier, 2014; Wendling y Souza Junior, 2003). Se demuestra así, la viabilidad técnica de la propagación vegetativa, por minicepas y miniestacas de yerba mate. No obstante, a la fecha, no existe un protocolo operativo factible de ser aplicado por pequeños y medianos viveristas regionales, como el descripto en el presente trabajo.

\section{Grado de demanda:}

El protocolo descripto en el presente trabajo es una herramienta tecnológica que permitirá maximizar el uso del espacio en el vivero, aumentando la tasa de multiplicación y homogeneidad en el manejo de vivero. Este protocolo es el resultado de un proyecto de investigación financiado por el Instituto Nacional de la Yerba Mate (INYM), la Facultad de Ciencias Forestales y la Universidad Nacional de Misiones, que surgió como demanda de productores locales de yerba mate, a partir de talleres participativos realizado por el INYM, e incluido en su plan estratégico.

El objetivo del proyecto fue desarrollar una metodología de propagación vegetativa de yerba mate, mediante la técnica de minicepas y miniestacas. Generando así 
Innovación y Desarrollo Tecnológico y Social (2019) 1 (1): 61-72

tecnologías que maximicen el uso del espacio en el vivero, aumenten la tasa de multiplicación y homogeneidad en el manejo de vivero.

Para llevar adelante este objetivo general se plantearon los siguientes objetivos específicos:

> Estudiar el efecto del manejo de minicepas en la capacidad de brotación, número de miniestacas útiles y la capacidad de enraizamiento de las miniestacas obtenidas de las minicepas.

> Determinar el manejo de miniestacas más apropiado para producir el mayor porcentaje de enraizamiento, y la mejor calidad de planta a obtener, como así también el tiempo requerido.

\section{Desarrollo del producto}

Manejo de Minicepas

Material vegetal: se recomienda comenzar a trabajar con plantines de 6-8 meses, que presenten un diámetro al cuello de $3-4 \mathrm{~mm}$ y una altura mayor a $20 \mathrm{~cm}$.
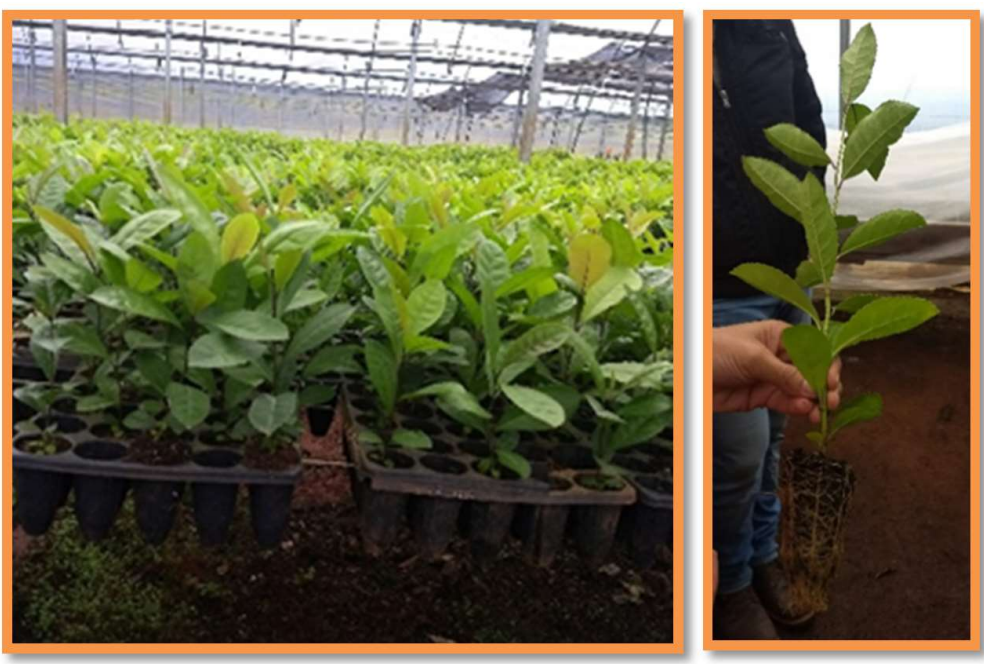

Figura 1. Morfologia de los plantines / Figure 1. Seedlings morphology

Formación de las minicepas (Plantas madre donantes de brotes-estacas): los plantines deben ser trasplantados a macetas sopladas de tamaño $\mathrm{N}^{\circ} 120$ 14, conteniendo sustrato de corteza de pino compostada, y fertilizante de liberación lenta, por ejemplo N:14-P:9-K:15-2MgO + micro de 5 a 7 meses de liberación o N:13-P:6$\mathrm{K}: 16$ + micro) de 6 meses de liberación; en una concentración de $3 \mathrm{~kg} / \mathrm{m}^{3}$ de sustrato. 
Innovación y Desarrollo Tecnológico y Social (2019) 1 (1): 61-72

Decapitación de las minicepas: A los 15 días del trasplante, se puede proceder a la decapitación de los plantines, recomendando realizar esta operación a fines de agosto, principio de setiembre. Este procedimiento consiste en remover el ápice del plantín, con tijera corta papeles o de podar desinfectada con alcohol etílico al $70 \%$, procurando dejar una porción de 4-8 cm desde el cuello de la planta, para incentivar el rebrote. Se recomienda efectuar una aplicación con spray de una solución de fungicida (EtilenbisDitiocarbamato de Zinc) de $3 \mathrm{~g} / \mathrm{L}$, para prevenir infecciones.
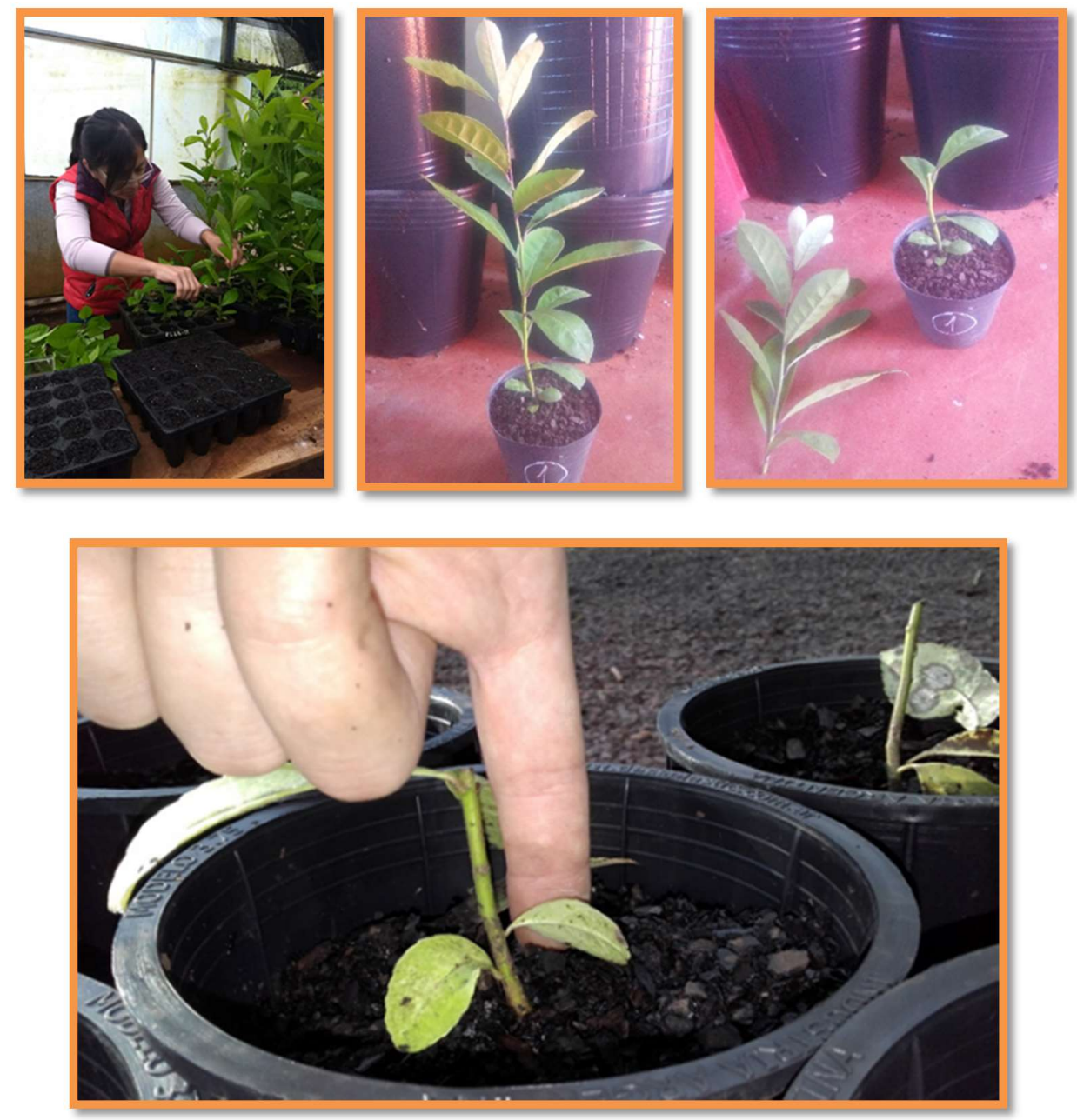

Figura 2. Decapitacion y manejo de las minicepas / Figure 2. Stock plant dettoping and management

Condiciones de cultivo de las minicepas: Las minicepas deben ser cultivadas en invernáculo de polietileno, con una media-sombra del $30 \%$, con riego por goteo. En una frecuencia de riego cada 60-90 minutos, por 5 minutos; desde las 9-10 hs de la mañana hasta las 17-18 hs de la tarde, dependiendo de la estación del año. 
Innovación y Desarrollo Tecnológico y Social (2019) 1 (1): 61-72

Un aspecto fundamental en el cultivo de las minicepas es la luz necesaria para que la planta tenga capacidad fotosintética suficiente para acumular carbohidratos en los brotes, de manera de obtener una tasa de producción de estacas útiles con una alta capacidad de enraizamiento. Por otro lado, el riego por goteo impedirá el contacto de los brotes con el agua disminuyendo significativamente la posterior pudrición de las estacas en el proceso de enraizamiento.
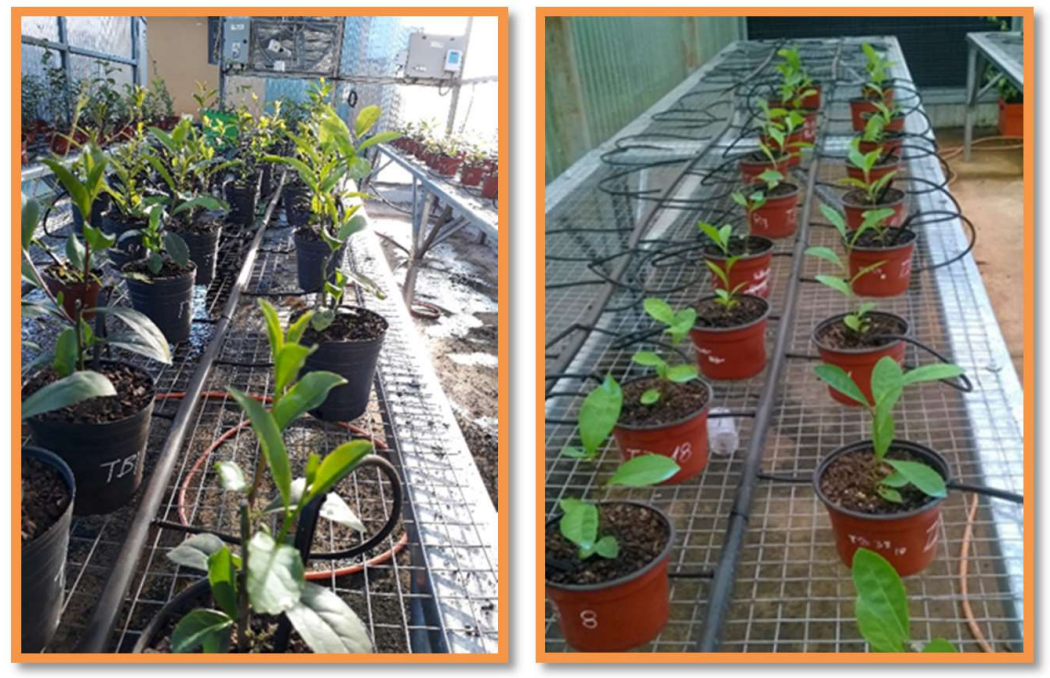

Figura 3. Condiciones de cultivo de lasminicepas / Figure 3. Stock plant growth conditions

\section{Cosecha de brotes y manejo de las miniestacas}

Cuando los brotes han alcanzado la longitud y el diámetro apropiado para la obtención de miniestacas útiles(entre 45 y 60 días dependiendo de la época del año) se procede a la cosecha de estos.

Cosecha de brotes: En el invernáculo, se cosechan los brotes con tijera corta papeles o de podar, previamente desinfectada en alcohol etílico al 70\%. Inmediatamente de cortados, se deben colocar en un recipiente con agua, de forma que las bases permanezcan constantemente hidratadas. 

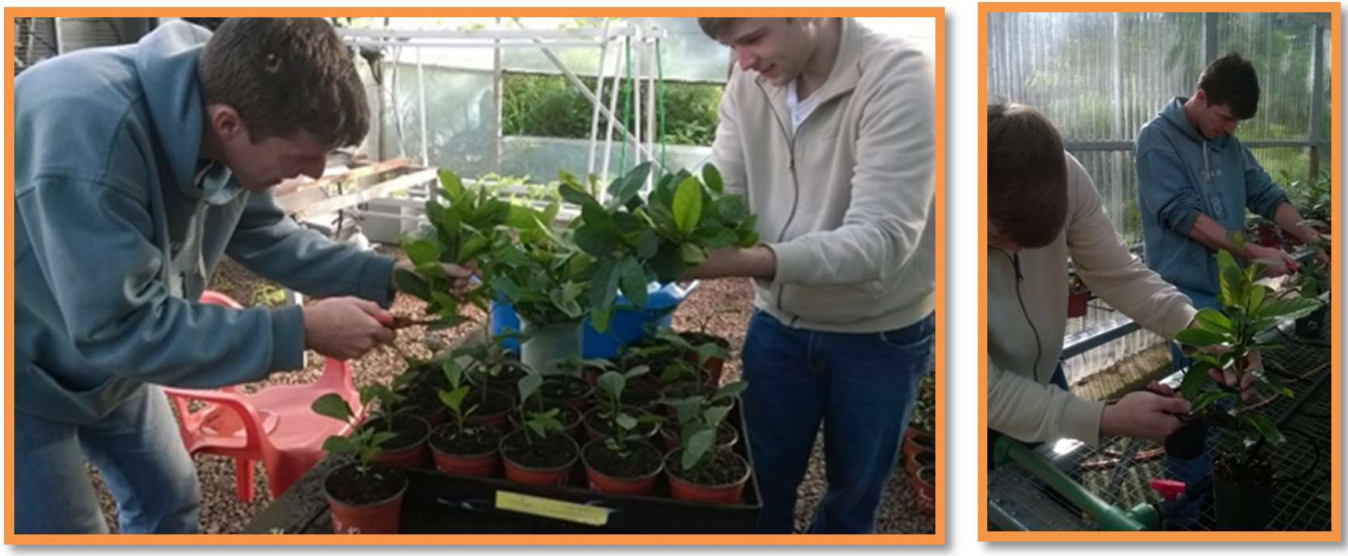

Figura 4. Cosecha de brotes / Figure 4. Shoot harvest

Manejo y preparación de las miniestacas: Una vez cosechados los brotes, se comienza el procesamiento en el lugar apropiado, definido por el vivero, que cuente con una mesa o mesada de trabajo. Los brotes son divididos en miniestacas primarias (que contienen el ápice), secundarias (la miniestaca de segundo orden) y/o terciarias (de tercer orden). Se procura dejar una miniestaca de $5 \mathrm{~cm}$ de longitud, entre 3-4 mm de diámetro, y que contenga 2 nudos o yemas, considerándose a ésta como "miniestaca útil". Se procede a cortar las hojas a la mitad, de manera de evitar el exceso de transpiración, pero que quede suficiente superficie de hoja para que pueda continuar fotosintetizando y generando carbohidratos necesarios para el enraizamiento. A medida que se procesan las miniestacas, se colocan las bases de las mismas en agua para evitar la desecación, que impediría el posterior enraizamiento. 
Innovación y Desarrollo Tecnológico y Social (2019) 1 (1): 61-72
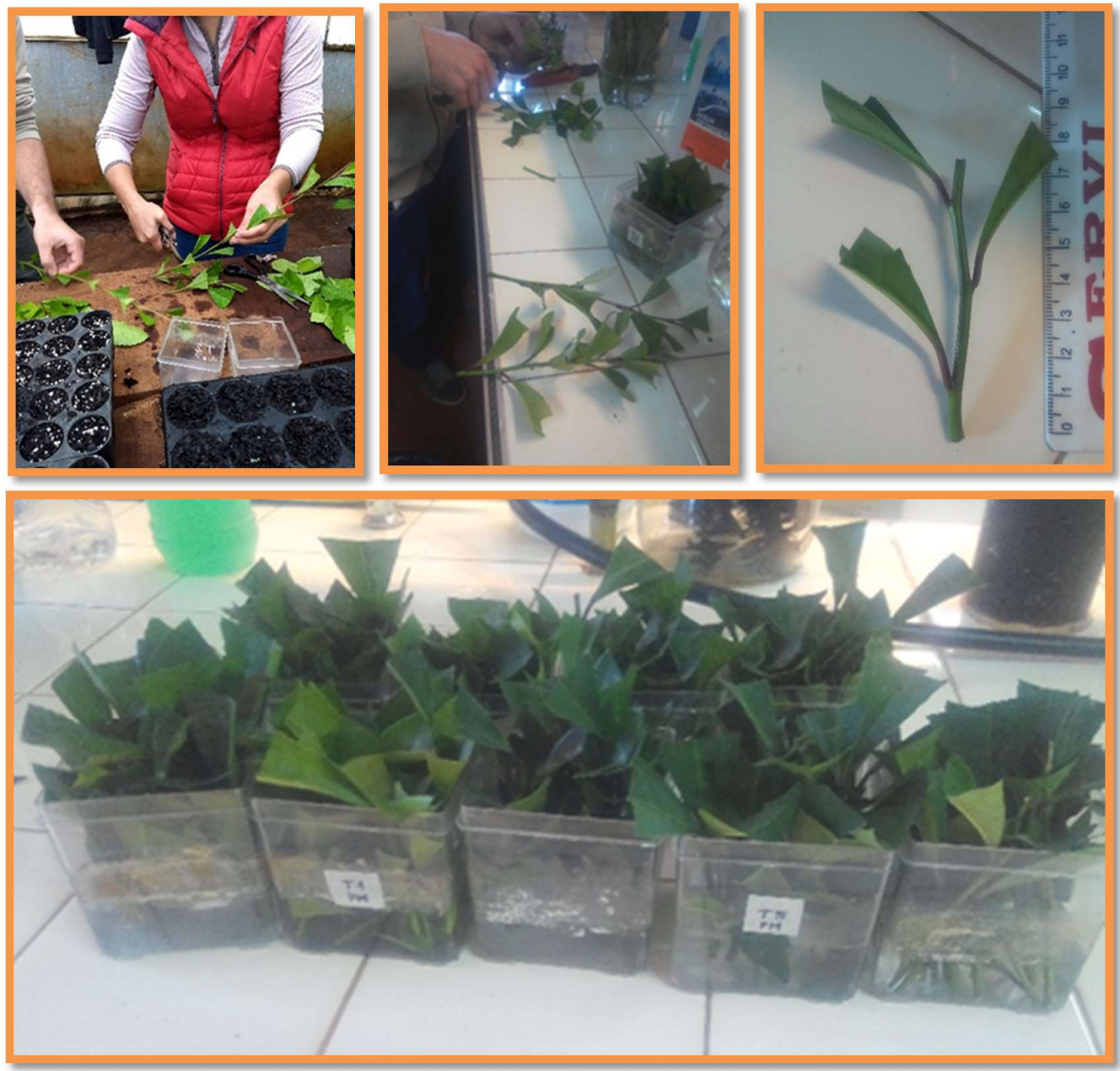

Figura 5. Manejo y preparación de las miniestacas / Figure 5. Handling and preparation of minicuttings

Tratamiento preventivo de las miniestacas: Una vez procesadas las miniestacas, se procede a colocar las mismas en una solución de fungicida Etilenbis-Ditiocarbamato de Zinc en una concentración de $20 \mathrm{~g} / \mathrm{L}$, por 20 minutos, como tratamiento preventivo para evitar la pudrición de las miniestacas en el proceso de enraizamiento. 


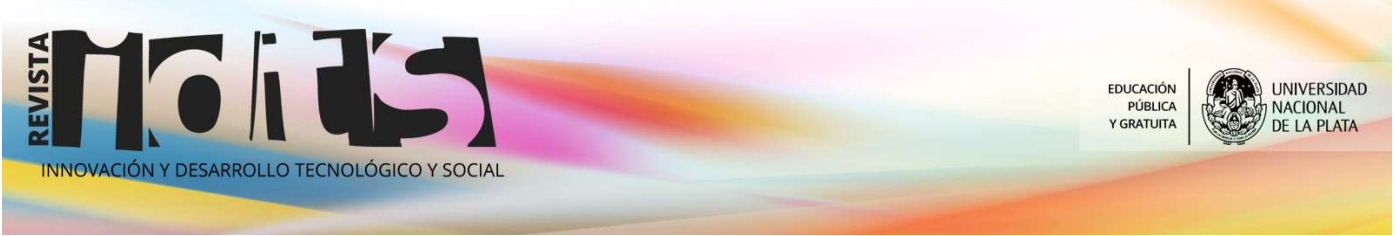

Innovación y Desarrollo Tecnológico y Social (2019) 1 (1): 61-72

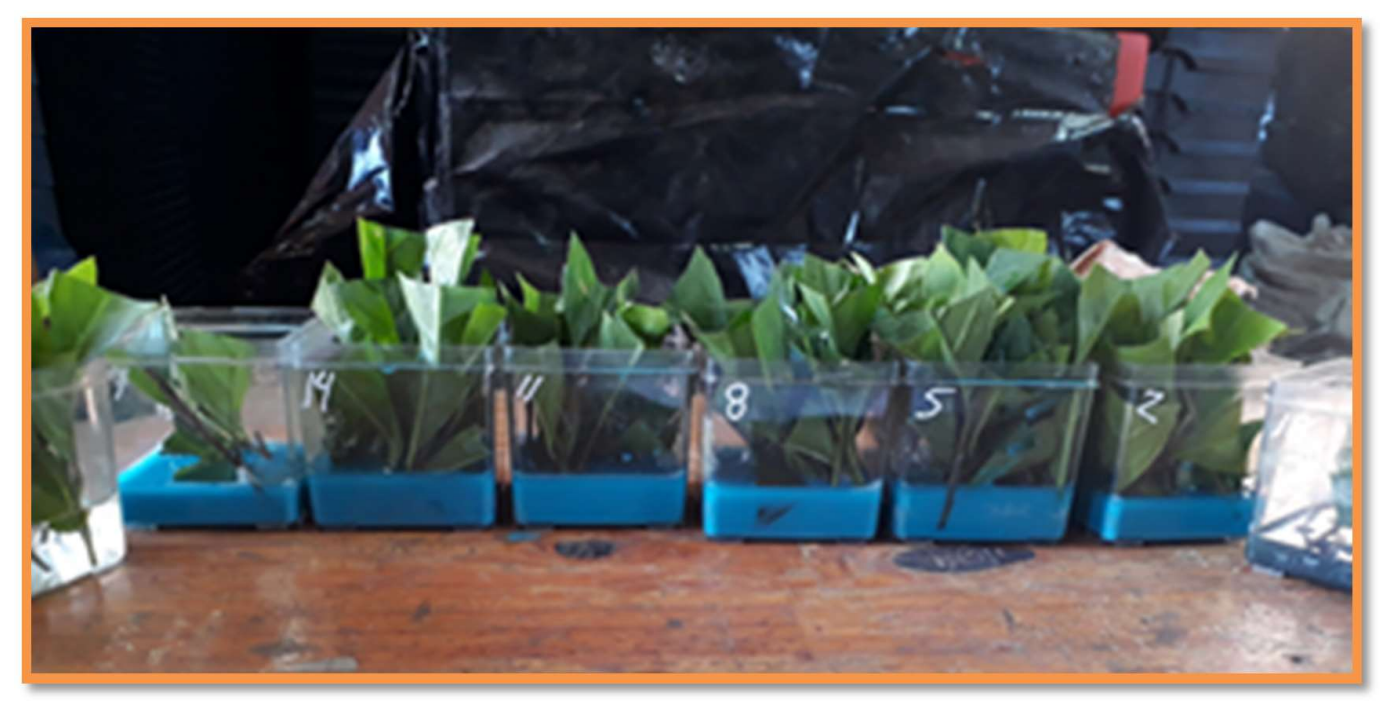

Figura 6. Pre-tratamiento de las miniestacas con fungicida / Figure 6. Minicuttings fungicidepretreatment

Época de cosecha: Las fechas apropiadas para la cosecha y el enraizamiento de las miniestacas es noviembre y diciembre, de forma de llegar con la planta lista para plantación en mayo-junio. Se podría realizar otra cosecha en enero o febrero, para plantaciones tardías.

\section{Enraizamiento de las miniestacas.}

Una vez transcurridos los 20 minutos del pretratamiento con la solución de fungicida, las miniestacas deben ser trasplantadas al sustrato.

Bandejas, sustratos y fertilizante: Se pueden utilizar las bandejas de $90 \mathrm{~cm}^{3}$, o las descartables de 25 cavidades, comúnmente utilizadas para plantines de yerba. Las bandejas deben ser rellenadas con el sustrato correspondiente:

Corteza de pino compostada: Recomendado en caso de que se tenga un muy buen control del riego y humedad, que no sea excesivo.

Corteza de pino + perlita: En una relación 3 de corteza y 1 de perlita, en caso de que no se tenga buen control del riego. La perlita previene la compactación, y proporciona una excelente relación de aireación: retención.

Fertilizante: Se recomienda utilizar un fertilizante de base de liberación lenta $\left(3 \mathrm{~kg} / \mathrm{m}^{3}\right)$. Una vez logrado el enraizamiento, se procede a la aplicación de fertilizante foliar, de acuerdo con los que se hayan utilizado en el manejo de los plantines. 
Innovación y Desarrollo Tecnológico y Social (2019) 1 (1): 61-72

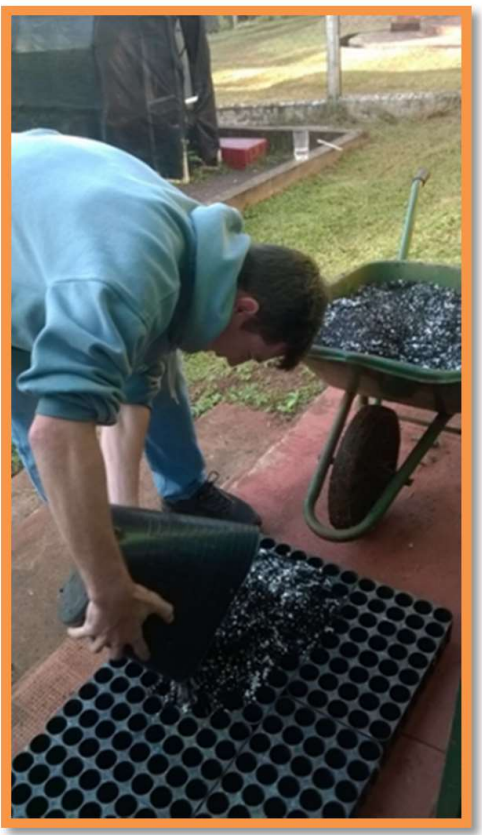

Figura 7. Preparación del sustrato / Figure 7. Substrate preparation

Inserción de la miniestaca en el sustrato: Se recomienda insertar $1 / 3$ de la base de las miniestacas en el sustrato. Para esto, se debe contar con un palillo o bolígrafo en desuso, para hacer primero los agujeros en el sustrato, en el cual se va a insertar la miniestaca. Asegurarse de presionar bien alrededor de la miniestaca, para que la misma no quede floja y se descalce cuando reciba el riego. A los 15 días de establecida la miniestaca, se recomienda asperjar con una solución de fungicida EtilenbisDitiocarbamato de Zinc (3g/L)
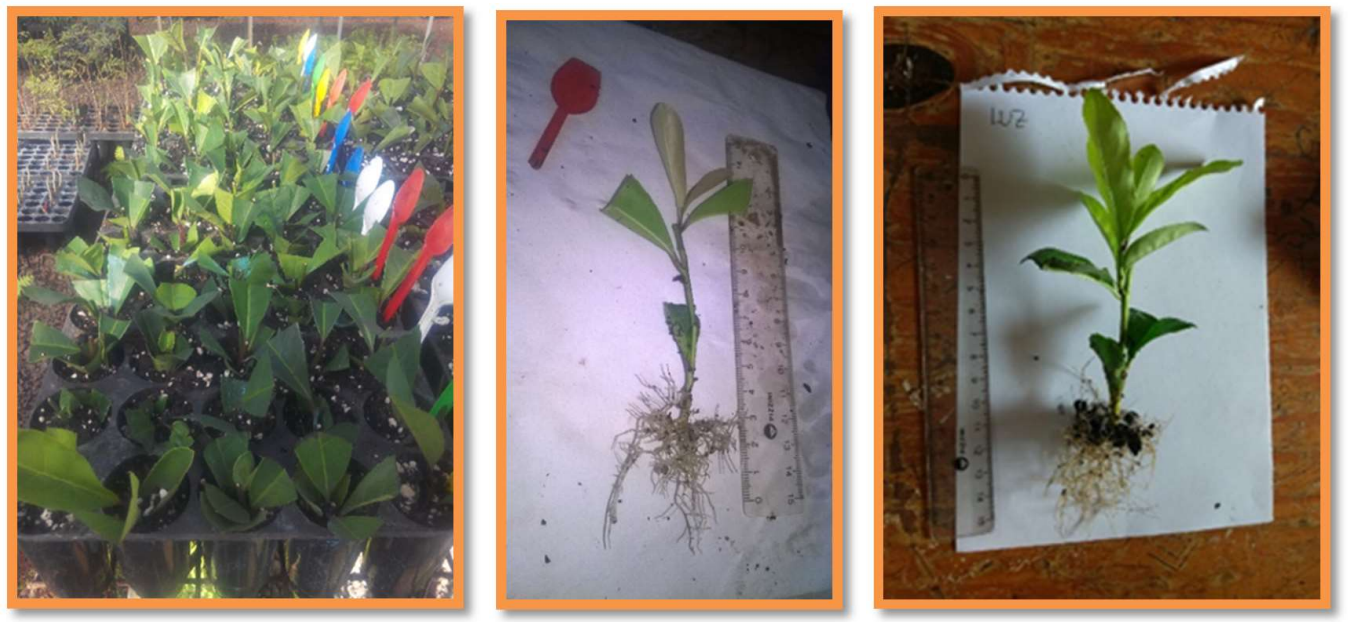

Figura 8. Miniestacas en proceso de enraizamieto y miniestacas enraizadas / Figure 8. Minicuttingsin the process of rooting and rooted minicuttings 


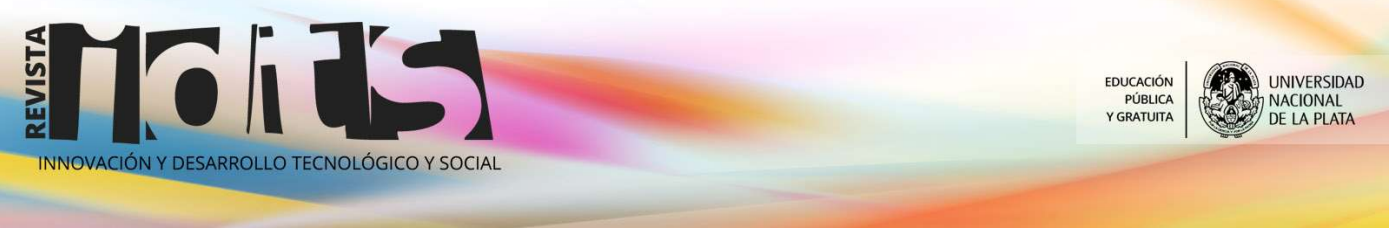

Innovación y Desarrollo Tecnológico y Social (2019) 1 (1): 61-72

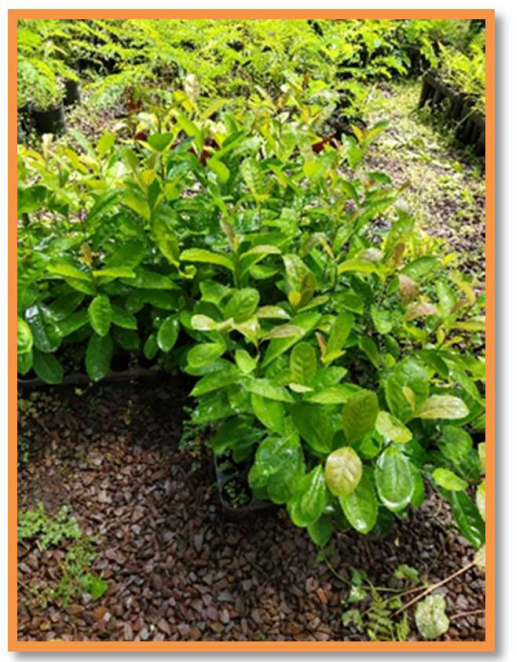

Figura 9.Plantines logrados a los 5 meses de establecidos en enraizamiento / Figure 9. Planlets achieved after 5 months on rooting.

Riego: Se recomienda el riego por microaspersión, con $8 \mu \mathrm{m}$ (boquilla negra, con antigoteo), con una frecuencia de riego cada 30 minutos, por 90 segundos de riego, desde las 9-10 hs de la mañana, hasta las $17-18$ hs de la tarde, dependiendo de la estación del año (la acumulación de agua promedio en 24 hs puede variar de 3-6 $\mathrm{mm} / \mathrm{m}^{2}$ ). Es importante el control diario del riego, aunque este sea automático, porque los días de lluvia o excesiva humedad, las condiciones en el interior del invernáculo cambian, y en algunos casos puede ser necesario desconectar el riego por algunas horas.

Condiciones de cultivo: Invernáculo de polietileno, con una humedad recomendable de un $70-90 \%$, y media sombra de $30-50 \%$.

Tiempo promedio de enraizamiento: 45 y 60 días.

\section{TASA DE MULTIPLICACIÓN}

De los estudios realizados, en el desarrollo del proyecto, se pudieron determinar las siguientes tasas de producción:

Producción de miniestacas útiles/planta $\rightarrow$ septiembre (4 miniestacas/planta); diciembre (7 miniestacas/planta) y febrero (6 miniestacas/planta). Producción total de 17 miniestacas/planta/año 


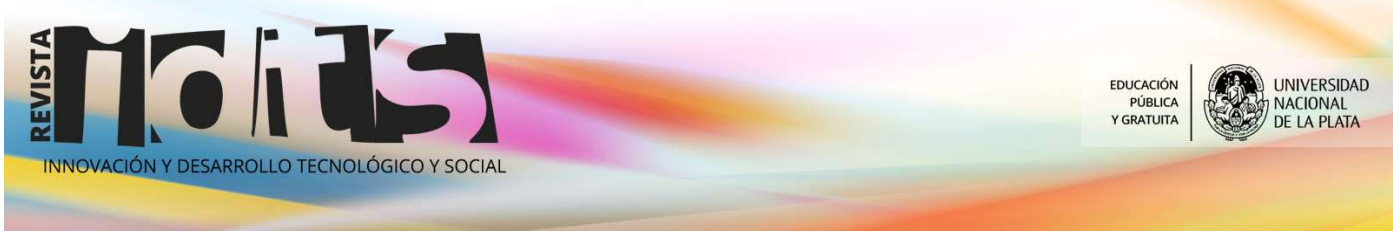

Innovación y Desarrollo Tecnológico y Social (2019) 1 (1): 61-72

Producción promedio de miniestacas útiles $/ \mathrm{m}^{2}\left(50\right.$ macetas de $\left.1 \mathrm{~L} / \mathrm{m}^{2}\right)$ por cosecha $\rightarrow$ 285

Producción promedio de miniestacas útiles/ $\mathrm{m}^{2} /$ año $\rightarrow 855$

Porcentaje de enraizamiento $\rightarrow(70-85 \%) 80 \%$

Producción de plantas enraizadas $/ \mathrm{m}^{2}$ de minicepas/año $\rightarrow 684$

Duración estimada de la minicepa (sin efecto del envejecimiento en la capacidad de enraizamiento) $\rightarrow$ de al menos 4 años (estudio en proceso)

\section{Información sobre el patentamiento/registro de la innovación o desarrollo}

El presente desarrollo es delibre uso, no ha sido patentado, y no presenta registro de propiedad.

\section{Financiamiento}

Este protocolo es el resultado de un proyecto de investigación financiado por el Instituto Nacional de la Yerba Mate (INYM), la Universidad Nacional de Misiones (UNaM), la Facultad de Ciencias Forestales (FCF, UNaM),y el Consejo Interuniversitario Nacional (CIN).

\section{Agradecimientos}

Al INYM por el financiamiento mediante un proyecto PRASY y becas de estudios. A la FCF-UNaM, por permitir el uso de equipos e infraestructura; y a la UNaM y el CIN por las becas de grado para alumnos participantes del proyecto.

A los becarios, estudiantes de la carrera de Ing. Agronómica (FCF-UNaM), Misael Naumann; Jimena Ríos; Jonathan Holtzmaisters; Máximo Petruk Nowosad; Walter Londero; Lucas Wedhen; Nadia Zajaczkowski, por su colaboración en el desarrollo del proyecto.

\section{Referencias bibliográficas}

Assis, T. F., y Mafia, R. (2007). Hibridação e clonagem. Biotecnologia florestal. (Suprema, Ed.).

Bitencourt, J., Zuffellato-Ribas, K. C., Wendling, I., y Koehler, H. S. (2009). Enraizamento de estacas de erva-mate (Ilex paraguariensis A. St.-Hill.) provenientes de brotações rejuvenescidas. Revista Brasileira de Plantas Medicinais, 11(3), 277-281.

Brondani, G. E., Araujo, M. A. de, Wendling, I., y Kratz, D. (2008). Enraizamento de miniestacas de Erva-Mate sob diferentes ambientes. Pesquisa Florestal Brasileira, o(57), 29-38.

Brondani, G. E., Wendling, I., Araújo, M. A. de, Santin, D., Benedetti, E. L., y Roveda, L. F. (2009). COMPOSIÇÕ̃ES DE SUBSTRATOS E AMBIENTES DE ENRAIZAMENTO NA 


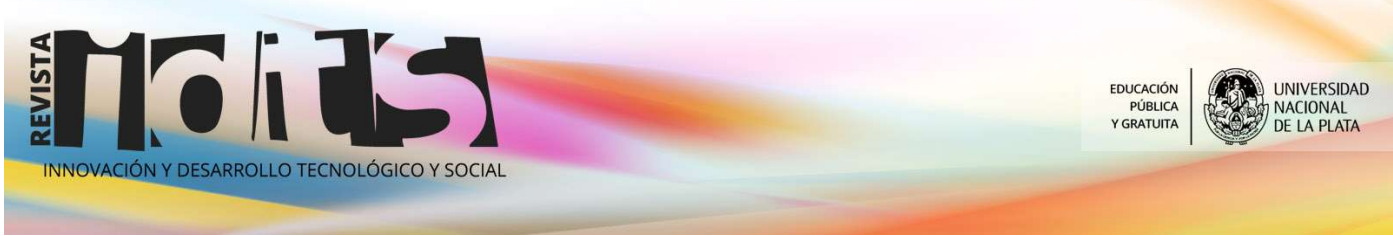

Innovación y Desarrollo Tecnológico y Social (2019) 1 (1): 61-72

ESTAQUIA DE Ilex paraguariensis A. St.-Hil. FLORESTA, 39(1), 41-49.

Eibl, B., Fernandez, R. A., Kozarik, J. C., Lupi, A., Montagnini, F., y Nozzi, D. (2000). Agroforestry systems with Ilex paraguariensis (American holly or yerba mate) and native timber trees on small farms in Misiones, Argentina. Agroforestry Systems, 48(1), 1-8.

Galíndez, G., Ceccato, D., Bubillo, R., Lindow-López, L., Malagrina, G., Ortega-Baes, P., y Baskin, C. C. (2018). Three levels of simple morphophysiological dormancy in seeds of Ilex (Aquifoliaceae) species from Argentina. Seed Science Research, 28(2), 131-139. https://doi.org/10.1017/S0960258518000132

INYM. (2016). Superficie cultivada por departamento. Recuperado de http://www.inym.org.ar/wp-content/uploads/2017/02/sup_cultivada_dpto.pdf

Naumann, M., Rocha, P., Duarte, E., Morales, V., y Niella, F. (2017). Estudio de factores que afectan la capacidad de enraizmiento de miniestacas de Ilex paraguiariensis St. Hill. Yvyrareta, 25, 15-20.

Niella, F., Rocha, P., Eibl, B., Schoffen, C., Martinez, M., Conti, P., ... Ayala, L. (2014a). Extendiendo los beneficios de las técnicas de propagación clonal a pequeños y medianos productores como estrategia de conservación y domesticación. En 16as Jornadas Técnicas Forestales y Ambientales (pp. 308-316). Eldorado: FCF - INTA.

Niella, F., Rocha, P., Eibl, B., Schoffen, C., Martinez, M., Conti, P., ... Ayala, L. (2014b). Propagación clonal de Peltophorum dubium (caña fistola), Myrocarpus frondosus (incienso), y Cordia trichotoma (peteribi) para su conservación y domesticación. Yvyrareta, 22, 15-20.

Pino, P. S., y Laviosa, G. (1998). Multiplicación vegetativa de yerba mate por estacas terminales con hojas. Investigación Agraria, 2(1), 28-31.

Prat Kricun, S. D. (1993). Yerba Mate Tecnicas actualizadas de cultivo. Cerro Azul: Instituto Nacional de Tecnologia Agropecuaria.

Ramirez, C., y Rocha, P. (2017). Efecto genotipo y manejo de la planta madre en la capacidad de enraizamiento para propagacion de Cedrela Fissilis (Cedro Misionero). Yvyrareta, 24, 36-44.

Rocha, P., Niella, F., Eibl, B., Schoffen, C., Martinez, M., Conti, P., ... Scherf, A. (2015). Desarrollo de protocolos de propagación vegetativa de especies leñosas, nativas y exóticas de la Provincia de Misiones. En Congreso Nacional de Viveros Cítricos, Forestales y Ornamentales (pp. 52-54). Posadas.

Wendling, I., Dutra, L., y Grossi, F. (2007). Produção e sobrevivência de miniestacas e minicepas de erva-mate cultivadas em sistema semi-hidropônico. Pesquisa Agropecuária Brasileira, 42(2), 289-292. 


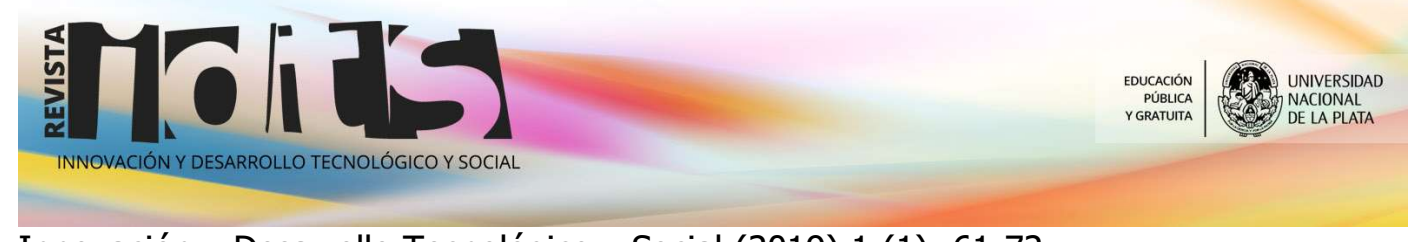

Innovación y Desarrollo Tecnológico y Social (2019) 1 (1): 61-72

Wendling, I., y Souza Junior, L. (2003). Propagação vegetativa de erva-mate (Ilex paraguariensis Saint Hilaire) por miniestaquia de material juvenil. En Congresso sulamericano da erva-mate.

Wendling, I., Trueman, S., y Xavier, A. (2014). Maturation and related aspects in clonal forestry-part II: reinvigoration, rejuvenation and juvenility maintenance. New Forests, $45(4), 473-486$. 